\title{
Association between alpha-fetoprotein and metabolic syndrome in a Chinese asymptomatic population: a cross-sectional study
}

Yimin Chen ${ }^{1+}$, Ying Zhao ${ }^{2 \dagger}$, Linmin Feng ${ }^{2}$, Jie Zhang ${ }^{2}$, Juanwen Zhang ${ }^{2}$ and Guofang Feng ${ }^{3^{*}}$

\begin{abstract}
Background: Metabolic syndrome is closely associated with an increased risk for fatty liver disease morbidity and mortality. Recently, studies have reported that participants with fatty liver disease have higher serum alpha-fetoprotein levels than those without. We investigated the association between alpha-fetoprotein levels and the prevalence of metabolic syndrome in a Chinese asymptomatic population.

Methods: A cross-sectional study was performed with 7755 participants who underwent individual health examinations. Clinical and anthropometric parameters were collected and serum alpha-fetoprotein levels and other clinical and laboratory parameters were measured. Logistic regression analysis was used to examine associations between alpha-fetoprotein and metabolic syndrome.

Results: Participants with metabolic syndrome had significantly higher $(p<0.001)$ alpha-fetoprotein levels than those without, though all alpha-fetoprotein levels were within the reference interval. The association between the components of metabolic syndrome (central obesity, elevated blood pressure, elevated triglycerides, reduced high-density lipoprotein cholesterol, and elevated fasting plasma glucose) and alpha-fetoprotein levels was evaluated. Alpha-fetoprotein levels in the elevated triglycerides, reduced high-density lipoprotein cholesterol, and elevated fasting plasma glucose groups were significantly different $(p=0.002, p<0.001, p=0.020$ ) compared with alpha-fetoprotein in the normal triglycerides, high-density lipoprotein cholesterol, and fasting plasma glucose groups. Logistic regression analyses showed an association between alpha-fetoprotein levels and increased risk for metabolic syndrome, the presence of reduced high-density lipoprotein cholesterol, and elevated fasting plasma glucose, but not with obesity, elevated blood pressure, or triglycerides.
\end{abstract}

Conclusions: These results suggest a significant association between alpha-fetoprotein and metabolic syndrome.

Keywords: Alpha-fetoprotein, Metabolic syndrome, Fatty liver disease

\section{Background}

Metabolic syndrome (MS) is characterized by a number of metabolic risk factors including abdominal obesity, impaired glucose metabolism, dyslipidemia, and hypertension [1-3]. MS is also associated with the development of diabetes mellitus, cardiovascular

\footnotetext{
* Correspondence: FBFGF2@126.com

'Equal contributors

${ }^{3}$ The Affiliated Women's Hospital of Zhejiang University School of Medicine, Xueshi Road \#1, Hangzhou 310006, China

Full list of author information is available at the end of the article
}

disease, and non-alcoholic fatty liver disease (NAFLD) $[1,4,5]$. The prevalence of MS is increasing worldwide. In China, Gu et al. [6] reported that the prevalence of MS was $9.8 \%$ in men and $17.8 \%$ in women in 2005. Zhou et al. [7] conducted a cross-sectional survey in 14 provinces in China and reported that the prevalence of MS was $22.1 \%$ in men and $25.8 \%$ in women in 2014. Insulin resistance (IR) and chronic inflammation appear to be central mechanisms underlying the pathophysiology of MS [8].

\section{Biomed Central}


Alpha-fetoprotein (AFP) is a single-stranded glycoprotein primarily produced by the fetal liver and yolk sac. The level of AFP declines rapidly after birth and remains at a low level throughout the life of a person in the normal population [9-11]. AFP levels are reactivated in liver regeneration and hepatocarcinogenesis, which occur in hepatocellular carcinoma, chronic liver disease, acute or chronic viral hepatitis, and gonadal tumors [12-14]. Elevated serum AFP levels have been used mainly to predict the development of hepatocellular carcinoma, including large tumor size, advanced or metastatic stages, portal vein thrombosis, and postoperative recurrence [15]. Recently, Xu et al. [16] investigated the association between serum AFP and fatty liver disease (FLD) in a population of 9800 people undergoing health examinations and found that participants with FLD had higher AFP levels than those without. Additionally, Babalı et al. [12] found that patients with NAFLD had higher AFP levels than those without. AFP levels become increasingly elevated as the grade of liver steatosis increases, which suggests that AFP level monitoring might help clinicians to treat NAFLD.

The presence of hepatic inflammation, steatosis, and/or fibrosis may be the underlying cause of increased serum AFP levels in FLD patients with severe fatty liver [12, 16]. Because MS is closely associated with an increased risk for FLD morbidity and mortality [16], we investigated the associations between serum AFP and the prevalence of MS in a Chinese asymptomatic population.

\section{Methods}

\section{Patients}

This study included 8695 consecutive participants (4 609 men and 4. 086 women) who underwent individual health examinations that included a physical examination and clinical laboratory tests at the Health Care Centre of the First Affiliated Hospital of Zhejiang University School of Medicine between April and October 2014. All participants underwent serum AFP and biochemical parameter analyses. Patients were excluded if they exhibited any of the following conditions: viral hepatitis $(n=403)$, autoimmune hepatitis $(n=6)$, renal insufficiency $(n=89)$, chronic liver disease $(n=105)$, cancer $(n=65)$, elevated tumor markers $(n=87)$, pregnancy $(n=50)$, and recent infection $(n=135)$. The remaining 7755 participants $(4209$ men and 3546 women) were enrolled in the study. Informed consent was obtained from all participants and the study was approved by the ethics committee of the First Affiliated Hospital of the Medical College of Zhejiang University of China.

\section{Clinical and anthropometric parameters}

Waist circumference (WC), weight, height, systolic blood pressure (SBP), and diastolic blood pressure (DBP) were measured. Body mass index (BMI) was calculated as weight in kilograms divided by height in meters squared. Those with alcohol consumption $>140 \mathrm{~g} /$ week for men and $>70 \mathrm{~g} /$ week for women were categorized as drinkers, and those who smoked one or more cigarettes daily for at least 6 months were categorized as smokers.

\section{Laboratory techniques}

Laboratory examinations were conducted in the morning after an overnight fast. Alanine aminotransferase (ALT), aspartate aminotransferase (AST), gamma-glutamyltransferase $(\gamma-\mathrm{GT})$, triglycerides (TG), total cholesterol (Tch), high-density lipoprotein cholesterol (HDL-c), low-density lipoprotein cholesterol (LDL-c), fasting plasma glucose (FPG), and creatinine $(\mathrm{Cr})$ were assessed using an automatic biochemical analyzer (Hitachi 7600; Hitachi, Tokyo, Japan) with $\mathrm{GmbH}$ reagents (Roche Diagnostics, GmbH, Mannheim, Germany). Hemoglobin A1c (HbA1c) was assessed using a high-performance liquid chromatography analyzer (G8-90SL; Tosoh Corp., Tokyo, Japan) with Tosoh reagents. Analysis of AFP was performed using an automated chemiluminescence analyzer (Architect ci8200; Abbott Laboratories, Abbott Park, IL) with Abbott reagents.

\section{Diagnostic criteria for MS}

According to the International Diabetes Federation guidelines [17], MS is defined as having three or more of the following components: (i) $\mathrm{WC}>90 \mathrm{~cm}$ for Chinese men and $>80 \mathrm{~cm}$ for Chinese women; BMI $\geq 25 \mathrm{~kg} / \mathrm{m}^{2}$; (ii) TG $\geq 1.7 \mathrm{mmol} / \mathrm{L}$, or taking specific treatment for lipid abnormality; HDL-c $<1.03 \mathrm{mmol} / \mathrm{L}$ for men and $<1.29 \mathrm{mmol} / \mathrm{L}$ for women; (iii) $\mathrm{SBP} \geq 130 \mathrm{mmHg}$, or DBP $\geq 85 \mathrm{mmHg}$, or treatment for previously diagnosed hypertension; (iv) FPG $\geq 5.6 \mathrm{mmol} / \mathrm{L}$ or previously diagnosed type 2 diabetes.

\section{Statistical analyses}

Statistical analyses were performed using SPSS version 16 (SPSS, Chicago, IL). Data that were normally distributed are reported as mean \pm standard deviation and data that had a skewed distribution are reported as median and range. Differences between two groups were analyzed using the Student's $t$-test or the Mann-Whitney $U$ test. Differences among multi-groups were analyzed using one-way analysis of variance or the KruskalWalls $\mathrm{H}$ test. Spearman correlation analysis was used to examine correlations between serum AFP levels and clinical and laboratory parameters. Univariable and multivariable logistic regression was used to examine associations between AFP and MS. All statistical tests were two-tailed and a $p$-value $<0.05$ was considered statistically significant. 


\section{Results}

\section{Clinical characteristics of participants}

Demographic and biochemical characteristics of study participants are shown in Table 1 . There were 7755 participants included in the study and 1512 (19.5\%) fulfilled diagnostic criteria for MS. Patients with MS had significant differences in age, WC, BMI, SBP, DBP, ALT, AST, $\gamma$-GT, TG, Tch, HDL-c, LDL-c, FPG, HbA1c, Cr, AFP, prevalence of drinking, and FLD compared with patients without MS. The characteristics of all participants according to the number of components of MS are shown in Table 2. We also found significant differences in WC, BMI, SBP, DBP, ALT, AST, $\gamma$-GT, TG, Tch, HDL-c, LDL-c, FPG, HbA1c, Cr, AFP, prevalence of drinking, and FLD among these five groups. Participants with MS in groups with three and four MS components had higher age, WC, BMI, SBP, DBP, ALT, AST, $\gamma$-GT, TG, Tch, FPG, LDL-c, HbA1c, Cr, AFP, prevalence of drinking, and FLD, and lower HDL-c compared with patients in groups without MS or with 0-2 MS components. Notably, in groups MS 0 to MS 4, there were

Table 1 Demographic and biochemical characteristics of the study participants

\begin{tabular}{|c|c|c|c|}
\hline Variable & Without MS & With MS & $P$ value \\
\hline $\mathrm{n}$ & 6243 & 1512 & \\
\hline Age $(y r)$ & $43(18-87)$ & $51(26-82)$ & $<0.001$ \\
\hline $\operatorname{Sex}(M / F)$ & $3387 / 2856$ & $822 / 690$ & 0.937 \\
\hline$W C(\mathrm{~cm})$ & $82(58-123)$ & $91(64-115)$ & $<0.001$ \\
\hline BMI $\left(\mathrm{kg} / \mathrm{m}^{2}\right)$ & $23.2(15.6-36.9)$ & $26.1(17.3-35.6)$ & $<0.001$ \\
\hline $\mathrm{SBP}(\mathrm{mmHg})$ & $123 \pm 17$ & $143 \pm 15$ & $<0.001$ \\
\hline $\mathrm{DBP}(\mathrm{mmHg})$ & $75 \pm 11$ & $87 \pm 10$ & $<0.001$ \\
\hline ALT (U/L) & $18(4-188)$ & $24(4-193)$ & $<0.001$ \\
\hline AST (U/L) & $18(4-188)$ & $21(7-127)$ & $<0.001$ \\
\hline Y-GT (U/L) & $21(3-429)$ & 34 (8-816) & $<0.001$ \\
\hline TG $(m m o l / L)$ & $1.13(0.3-17.1)$ & $1.99(0.38-16.09)$ & $<0.001$ \\
\hline Tch (mmol/L) & $4.69(2.02-10.3)$ & $4.92(2.62-9.0)$ & $<0.001$ \\
\hline $\mathrm{HDL}-\mathrm{c}(\mathrm{mmol} / \mathrm{L})$ & $1.27(0.6-2.9)$ & $1.02(0.6-2.4)$ & $<0.001$ \\
\hline LDL-c (mmol/L) & $2.59(0.75-6.76)$ & $2.66(1-6.12)$ & $<0.001$ \\
\hline FPG (mmol/L) & 4.78 (3.45-16) & $5.19(3.87-13)$ & $<0.001$ \\
\hline $\mathrm{Cr}(\mathrm{mmol} / \mathrm{L})$ & $67(29-119)$ & $74(36-118)$ & $<0.001$ \\
\hline $\mathrm{HbA1c}(\%)$ & $5.5(3.5-11.6)$ & $5.8(4.2-13)$ & $<0.001$ \\
\hline $\operatorname{AFP}(\mathrm{ng} / \mathrm{ml})$ & $2.4(0.5-19.1)$ & $2.7(0.8-16.6)$ & $<0.001$ \\
\hline Drinker (\%) & 16.0 & 29.6 & $<0.001$ \\
\hline Smoker (\%) & 27 & 25.7 & 0.314 \\
\hline FLD (\%) & 29.0 & 62.3 & $<0.001$ \\
\hline
\end{tabular}

WC waist circumference, SBP systolic blood pressure, DBP diastolic blood pressure, $B M I$, body mass index, $A L T$ alanine aminotransferase, $A S T$ aspartate aminotransferase, $\gamma$-GT gamma-glutamyltransferase, TG triglycerides, Tch total cholesterol, $H D L-c$ high-density lipoprotein cholesterol, $L D L-c$ low-density lipoprotein cholesterol, FPG fasting plasma glucose, $\mathrm{Cr}$ creatinine, $\mathrm{HbA1c}$ hemoglobin A1c, AFP alpha-fetoprotein, FLD fatty liver disease significantly higher AFP levels observed in participants with MS $(p<0.001)$, although AFP levels were within the normal laboratory reference interval $(0-20 \mathrm{ng} / \mathrm{mL})$.

\section{Association between MS and serum AFP levels}

Serum AFP levels significantly correlated with age, WC, ALT, AST, $\gamma$-GT, HDL-c, LDL-c, TG, and Tch $(r=-0.087$, $0.121,0.058,0.085,0.218,0.113,0.085,0.232$, and 0.188 , respectively; all $p<0.05$ ) in participants with MS. In participants without MS, AFP significantly correlated with ALT, AST, $\gamma$-GT, LDL-c, TG, and Tch $(r=0.121,0.105$, $0.212,0.131,0.137$, and 0.162 , respectively; all $p<0.05$ ).

The components of MS included central obesity, elevated blood pressure, elevated TG, reduced HDL-c, and elevated FPG. The association between the components of MS and serum AFP levels in participants with and without MS are shown in Figs. 1 and 2. AFP levels in elevated TG, reduced HDL-c, and elevated FPG groups were significantly different compared with AFP in normal TG, HDL-c, and FPG groups in all participants.

Participants were divided into 10 groups according to deciles of serum AFP levels. The association between the 10 groups and the prevalence of MS are shown in Fig. 3. Across increasing serum AFP deciles, the prevalence of MS increased $(p<0.001)$.

\section{Risk factors for the presence of MS}

Univariate and multivariate logistic regression analyses was used to analyze the risk factors for MS in these asymptomatic participants. In the univariate regression model, serum AFP levels were associated with an increased risk for MS (odds ratio (OR): 1.072, $95 \%$ confidence interval (95 \% CI): 1.037-1.107, $p<0.001$; Table 3). After adjusting for age, WC, BMI, SBP, DBP, ALT, AST, $\gamma$-GT, TG, Tch, HDL-c, LDL-c, FPG, HbA1c, Cr, prevalence of drinking, and FLD using multivariate logistic analysis, AFP levels were also associated with an increased risk for MS (OR: 1.066, $95 \%$ CI: $1.007-1.129, p=0.028$; Table 3). The association between AFP and the presence of components of MS in the asymptomatic participants was also analyzed. The results did not show an association between AFP levels and the presence of central obesity (OR: 1.009, 95 \% CI: 0.958-1.062, $p=0.743)$, elevated blood pressure (OR: 1.032, $95 \%$ CI: 0.992-1.073, $p=0.119$ ), or elevated TG (OR: 1.02, 95 \% CI: 0.98-1.062, $p=0.338$ ). However, AFP levels were associated with the presence of reduced HDL-c (OR: 0.946, 95 \% CI: 0.9070.986, $p=0.008$ ) and elevated FPG (OR: 1.053, $95 \%$ CI: $1.012-1.096, p=0.010)$.

Analysis of the association between higher AFP (>3.4 ng/mL) and the prevalence of MS and its components is shown in Fig. 4. The 7755 participants were divided into two groups according to the $75 \%$ quartile 
Table 2 Clinical characteristics of the study participants grouped by the number of metabolic syndrome components

\begin{tabular}{|c|c|c|c|c|c|c|}
\hline \multirow[t]{2}{*}{ Variable } & \multicolumn{5}{|c|}{ No of components of MS } & \multirow[t]{2}{*}{$P$ value } \\
\hline & MS 0 & MS 1 & MS 2 & MS 3 & MS 4 & \\
\hline$n$ & 1335 & 2598 & 2310 & 1179 & 333 & $<0.001$ \\
\hline Age (yr) & $43(18-78)$ & $44(18-87)$ & $47(18-82)$ & $51(26-79) \#^{*} \$$ & $52(31-82) \#^{*} \$ \Delta$ & $<0.001$ \\
\hline $\operatorname{Sex}(M / F)$ & $735 / 600$ & $1296 / 1302$ & $1356 / 954$ & $621 / 558 \$$ & $201 / 132^{*} \Delta$ & $<0.001$ \\
\hline WC (cm) & 77 (58-90) & $80(60-123)$ & $88(60-116)$ & $90(64-115) \#^{*} \$$ & $92(81-115) \#^{*} \$ \Delta$ & $<0.001$ \\
\hline BMI (kg/m2) & $21.6(15.6-25)$ & $22.7(15.9-36.9)$ & $25.1(17.4-36.7)$ & $26.1(17.3-35.6) \#^{*} \$$ & $26.3(22.9-32.3) \# * \$ \Delta$ & $<0.001$ \\
\hline SBP $(\mathrm{mmHg})$ & $113 \pm 9$ & $119 \pm 14$ & $132 \pm 17$ & $140 \pm 15 \#^{*} \$$ & $150 \pm 14 \#^{*} \$ \Delta$ & $<0.001$ \\
\hline $\mathrm{DBP}(\mathrm{mmHg})$ & $69 \pm 7$ & $73 \pm 10$ & $81 \pm 12$ & $86 \pm 10 \#^{*} \$$ & $90 \pm 9 \#^{*} \$ \Delta$ & $<0.001$ \\
\hline ALT (U/L) & $15(4-69)$ & $17(5-188)$ & $20(4-151)$ & $24(4-123) \#^{*} \$$ & $30(10-193) \# * \$ \Delta$ & $<0.001$ \\
\hline AST (U/L) & $18(10-48)$ & $19(9-114)$ & $20(10-132)$ & $21(7-62) \#^{*} \$$ & $23(11-67) \#^{*} \$ \Delta$ & $<0.001$ \\
\hline Y-GT (U/L) & $16(3-377)$ & $20(5-306)$ & $24(4-429)$ & $31(8-816) \#^{*} \$$ & $50(10-485) \# * \$ \Delta$ & $<0.001$ \\
\hline TG $(m m o l / L)$ & $0.88(0.31-1.69)$ & $1.16(0.3-9.68)$ & $1.3(0.32-17.1)$ & $1.9(0.8-16.09) \#^{*} \$$ & $2.37(0.81-13.76) \#^{*} \$ \Delta$ & $<0.001$ \\
\hline Tch (mmol/L) & $4.65(2.92-7.89)$ & $4.69(2.13-8.49)$ & $4.71(2.02-10.3)$ & $4.86(2.62-9) \#^{*} \$$ & $5.18(2.8-8.23) \#^{*} \$ \Delta$ & $<0.001$ \\
\hline $\mathrm{HDL}-\mathrm{c}(\mathrm{mmol} / \mathrm{L})$ & $1.43(1.03-2.7)$ & $1.23(0.57-2.86)$ & $1.17(0.62-2.27)$ & $1.03(0.58-2.4) \#^{* \$}$ & $1.0(0.67-2) \#^{*} \$$ & $<0.001$ \\
\hline LDL-c (mmol/L) & $2.59(1.26-5.62)$ & $2.59(0.94-6.32)$ & $2.6(0.75-6.76)$ & $2.66(1-6.12) \#^{*} \$$ & $2.69(1.35-6.0) \#^{*} \$$ & 0.001 \\
\hline FPG (mmol/L) & $4.73(3.72-5.57)$ & $4.78(3.64-13)$ & $4.81(3.45-16)$ & $5(3.87-17) \#^{*} \$$ & $6(4.44-14) \#^{*} \$ \Delta$ & $<0.001$ \\
\hline $\mathrm{Cr}(\mathrm{mmol} / \mathrm{L})$ & $61(29-100)$ & $67(33-119)$ & $71(35-118)$ & $76(36-114) \#^{*} \$$ & $72(44-118)$ \#*\$ & $<0.001$ \\
\hline HbA1c (\%) & $5.5(3.5-6.1)$ & $5.5(4.4-10.8)$ & $5.6(4-11.6)$ & $5.7(4.2-13) \#^{*} \$$ & $6.4(4.7-13) \#^{*} \$ \Delta$ & $<0.001$ \\
\hline AFP (ng/ml) & $2.3(0.5-13.2)$ & $2.5(0.5-11.4)$ & $2.5(0.5-19.1)$ & $2.7(0.8-16.6) \#^{*} \$$ & $2.7(1.1-10.1) \#^{*} \$$ & $<0.001$ \\
\hline Drinker (\%) & 13.8 & 15.3 & 19.9 & $27.6 \# * \$$ & $37.0 \#^{*} \$ \Delta$ & $<0.001$ \\
\hline Smoker (\%) & 24.3 & 27.0 & 28.5 & 27.2 & 20.7 & 0.142 \\
\hline FLD (\%) & 12.1 & 22.5 & 45.5 & $63.6 \#^{*} \$$ & $60.7 \# * \$$ & $<0.001$ \\
\hline
\end{tabular}

$P$-value: comparison among these five groups; \# comparison vs. MS 0 group; * comparison vs. MS 1 group; $\$$ comparison vs. MS 2 group; $\triangle$ comparison vs. MS 3 group. WC waist circumference, SBP systolic blood pressure, DBP diastolic blood pressure, BMI body mass index, ALT Alanine aminotransferase, $A S T$ aspartate aminotransferase, $\gamma$-GT gamma-glutamyltransferase, $T G$ triglycerides, $T c h$ total cholesterol, $H D L$-c high-density lipoprotein cholesterol, $L D L$-c low-density lipoprotein cholesterol, FPG fasting plasma glucose, $\mathrm{Cr}$ creatinine, HbA1c hemoglobin A1c, AFP alpha-fetoprotein, FLD fatty liver disease

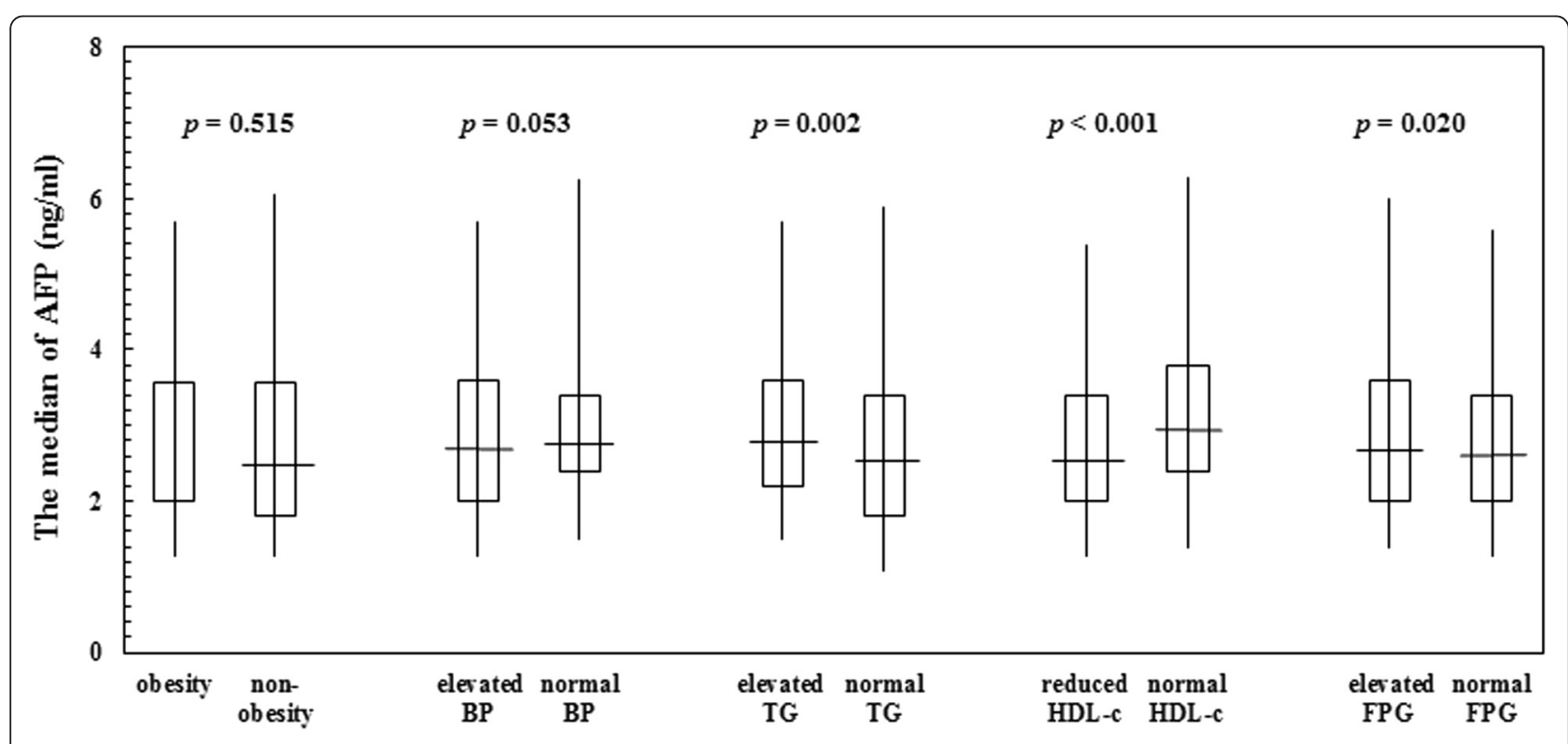

Fig. 1 Serum alpha-fetoprotein levels according to characteristics of metabolic syndrome in participants with metabolic syndrome 


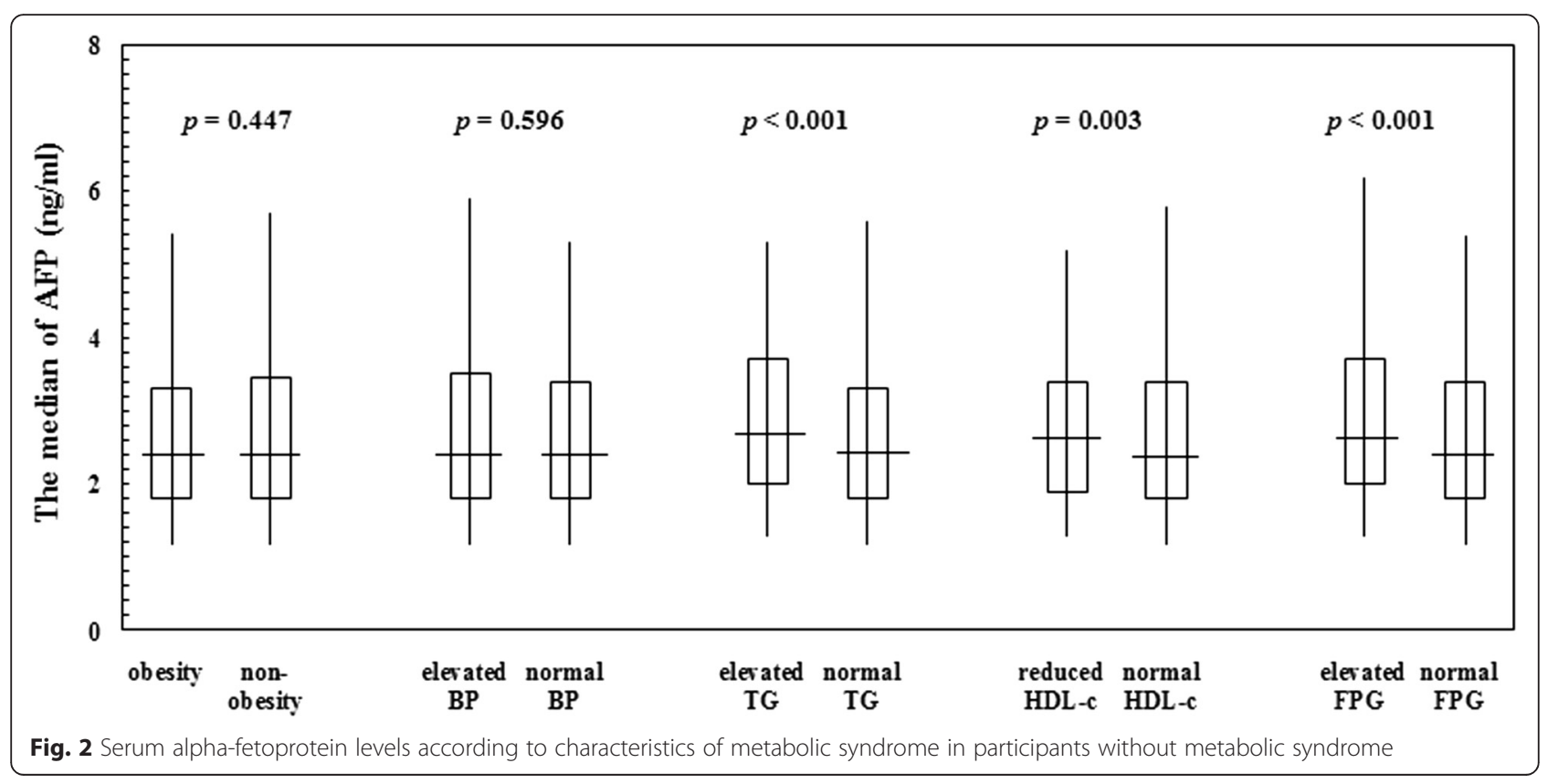

level of serum AFP (3.4 ng/mL). We observed a significantly higher prevalence of MS with the components including elevated BP, elevated TG, and elevated FPG in participants with AFP $>3.4 \mathrm{ng} / \mathrm{mL}$ compared with those with AFP $\leq 3.4 \mathrm{ng} / \mathrm{mL}$ (Fig. 4).

These results suggest that the presence of reduced HDL-c and elevated FPG may play a major role in the association between AFP and MS, while elevated BP and elevated TG may influence the association.

\section{Discussion}

In this study, serum AFP levels significantly correlated with WC, HDL-c, and TG $(p<0.001, p<0.001$, and $p<0.001$, respectively) in participants with MS.

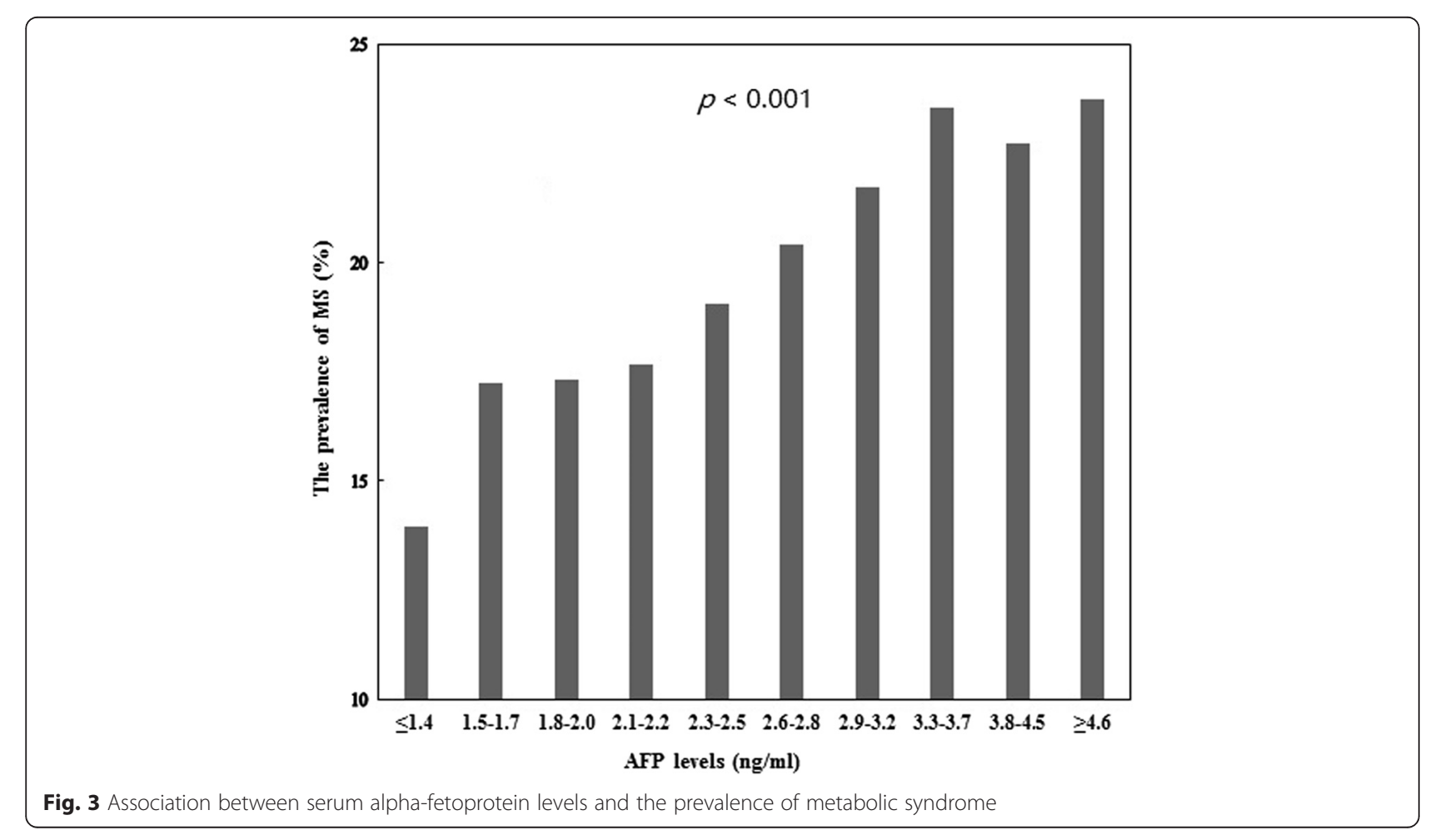


Table 3 Univariable and multivariable regression analyses for variables associated with metabolic syndrome

\begin{tabular}{|c|c|c|c|c|}
\hline \multirow[t]{2}{*}{ Variable } & \multicolumn{2}{|l|}{ univariable } & \multicolumn{2}{|l|}{ multivariable } \\
\hline & OR $(95 \% \mathrm{Cl})$ & $P$ value & OR $(95 \%$ Cl) & $P$ value \\
\hline Age $(y r)$ & $1.054(1.049-1.060)$ & $<0.001$ & $1.038(1.028-1.049)$ & $<0.001$ \\
\hline Sex (male) & $0.995(0.889-1.114)$ & 0.937 & & \\
\hline WC (cm) & $1.106(1.098-1.113)$ & $<0.001$ & $1.044(1.024-1.065)$ & $<0.001$ \\
\hline $\mathrm{BMI}\left(\mathrm{kg} / \mathrm{m}^{2}\right)$ & $1.345(1.318-1.373)$ & $<0.001$ & $1.124(1.062-1.190)$ & $<0.001$ \\
\hline $\mathrm{SBP}(\mathrm{mmHg})$ & $1.067(1.063-1.071)$ & $<0.001$ & $1.046(1.037-1.056)$ & $<0.001$ \\
\hline $\mathrm{DBP}(\mathrm{mmHg})$ & $1.094(1.088-1.100)$ & $<0.001$ & $1.027(1.013-1.041)$ & $<0.001$ \\
\hline $\operatorname{ALT}(\mathrm{U} / \mathrm{L})$ & $1.020(1.016-1.023)$ & $<0.001$ & 0.989 (0.980-0.998) & 0.016 \\
\hline AST (U/L) & $1.031(1.024-1.037)$ & $<0.001$ & $1.022(1.005-1.04)$ & 0.012 \\
\hline $\mathrm{Y}$-GT $(\mathrm{U} / \mathrm{L})$ & $1.010(1.009-1.012)$ & $<0.001$ & 1.005 (1.003-1.008) & $<0.001$ \\
\hline TG $(\mathrm{mmol} / \mathrm{L})$ & $1.712(1.63-1.798)$ & $<0.001$ & $1.192(1.071-1.326)$ & $<0.001$ \\
\hline Tch (mmol/L) & $1.377(1.297-1.462)$ & $<0.001$ & $1.082(0.816-1.433)$ & 0.585 \\
\hline $\mathrm{HDL}-\mathrm{c}(\mathrm{mmol} / \mathrm{L})$ & $0.057(0.045-0.073)$ & $<0.001$ & $0.032(0.02-0.052)$ & $<0.001$ \\
\hline LDL-c (mmol/L) & $1.226(1.133-1.325)$ & $<0.001$ & $1.081(0.777-1.504)$ & 0.644 \\
\hline FPG $(\mathrm{mmol} / \mathrm{L})$ & $1.971(1.845-2.105)$ & $<0.001$ & $1.722(1.477-2.008)$ & $<0.001$ \\
\hline $\mathrm{Cr}(\mathrm{mmol} / \mathrm{L})$ & $1.027(1.023-1.031)$ & $<0.001$ & $1.008(1.001-1.015)$ & 0.028 \\
\hline HbA1c (\%) & $2.815(2.506-3.162)$ & $<0.001$ & $1.600(1.278-2.004)$ & $<0.001$ \\
\hline $\operatorname{AFP}(n g / m l)$ & $1.072(1.037-1.107)$ & $<0.001$ & 1.066 (1.007-1.129) & 0.028 \\
\hline Drinker (yes) & $1.848(1.607-2.126)$ & $<0.001$ & $0.836(0.652-1.071)$ & 0.156 \\
\hline Smoker (yes) & $1.05(0.914-1.206)$ & 0.492 & & \\
\hline FLD (yes) & $4.051(3.602-4.556)$ & $<0.001$ & $1.496(1.210-1.849)$ & $<0.001$ \\
\hline
\end{tabular}

WC waist circumference, SBP systolic blood pressure, DBP diastolic blood pressure, $B M I$ body mass index, $A L T$ alanine aminotransferase, $A S T$ aspartate aminotransferase, $\gamma$-GT gamma-glutamyltransferase, $T G$ triglycerides, $T c h$ total cholesterol, $H D L-c$ high-density lipoprotein cholesterol, $L D L-c$ low-density lipoprotein cholesterol, FPG fasting plasma glucose, $\mathrm{Cr}$ creatinine, $\mathrm{HbA1c}$ hemoglobin A1c, AFP alpha-fetoprotein, FLD fatty liver disease

Participants with MS had significantly higher AFP levels $(p<0.001)$ than those without MS, although all AFP levels were within the reference interval. We evaluated the association between the components of MS (central obesity, elevated BP, elevated TG, reduced HDL-c, and elevated
FPG) and AFP levels, and found that AFP levels in the elevated TG, reduced HDL-c, and elevated FPG groups were significantly different $(p=0.002, p<0.001$, and $p=0.020)$ compared with AFP in the normal TG, HDL-c, and FPG groups. Furthermore, we found that in the higher AFP

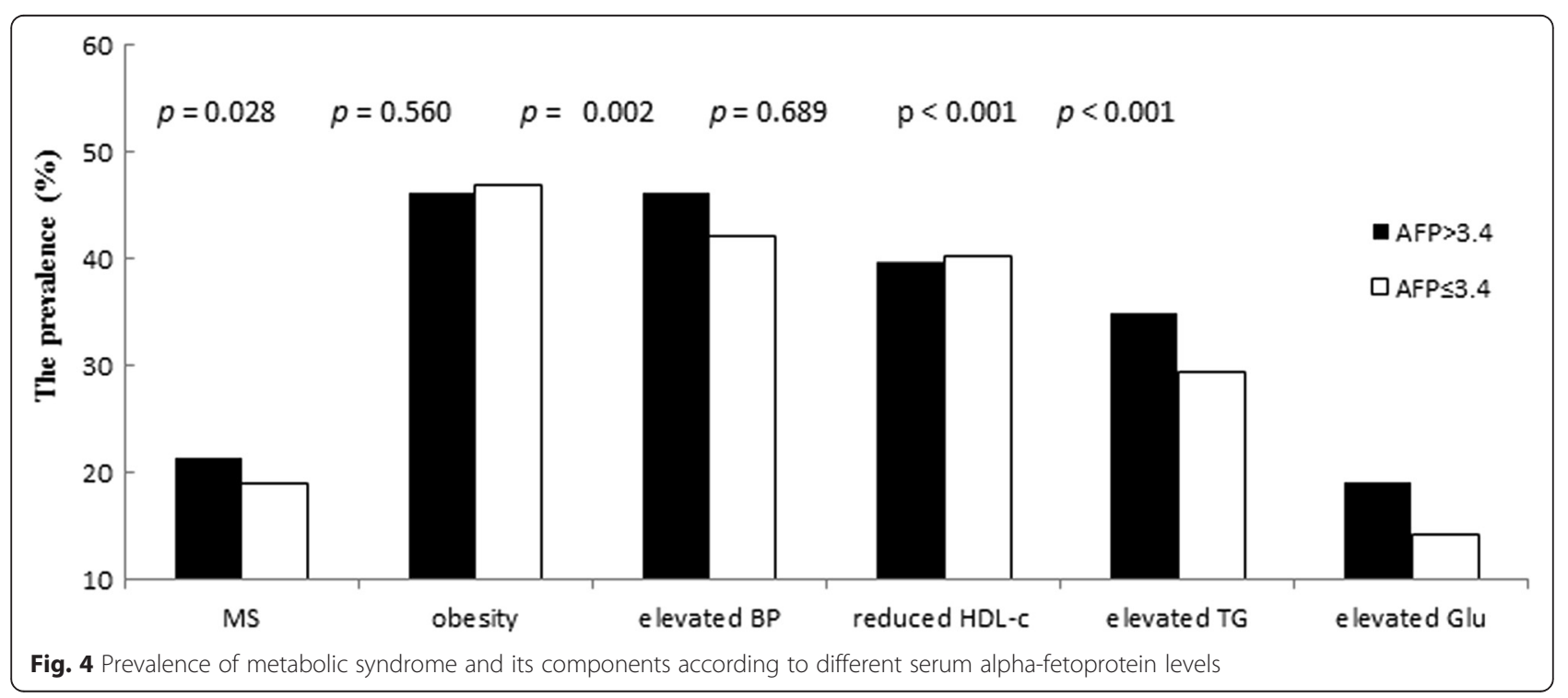


group ( $>3.4 \mathrm{ng} / \mathrm{mL}$ ), there was a higher prevalence of MS, elevated BP, elevated TG, and elevated FPG. Logistic regression analyses showed an association between AFP levels and increased risk for MS, reduced HDL-c, and elevated FPG, but no association between obesity, elevated BP, or TG. These results suggest a significant association between AFP and MS ( $p=0.028)$. Impaired glucose metabolism and dyslipidemia may play a major role in the association between AFP and MS.

Verhagen et al. [18] found that the prevalence of MS was highest in the IR group, and IR increased with the number of MS components. IR is associated with excessive fat accumulation in ectopic tissues, such as the liver, and plays a crucial role in the pathologic manifestations of MS, and is accompanied by elevated BP, elevated TG, reduced HDL-c, and impaired FPG [2, 19]. Some studies previously reported that FLD was more prevalent in patients with MS than in the general population, and FLD may be the hepatic component of MS because MS and FLD have a particularly close relationship [20-23].

The main potential mechanisms accounting for the association between MS and high AFP levels may be IR and hepatic steatosis. Hepatocytes play a major role in glucose homeostasis and can store or produce glucose depending on physical requirements [19]. IR impacts the hepatic glucose homeostatic pathways and leads to the release of free fatty acids from adipose tissue, elevates hepatic production of very-low-density lipoproteins, reduces high-density lipoproteins, and promotes inflammation and endoplasmic reticulum stress $[18,19]$. In a study with a large cohort, Porepa et al. [24] found newly diagnosed diabetes in adults with or without pre-existing hypertension, dyslipidemia, or obesity who appeared to be at higher risk for advanced liver disease than isolated hypertension, dyslipidemia, or obesity. In a study by Matsuzaka et al. [25], in which hepatic steatosis was associated with the development of IR, it was remained unclear whether IR leads to hepatic steatosis or whether steatosis enhances IR. Additionally, hepatic steatosis is associated with dyslipidemia and inflammatory markers $[20,21,25]$. Other studies have reported that increased chronic inflammation and oxidative stress in accumulated adipose tissue is an important pathogenic mechanism of obesity-associated MS [26-29]. Another potential mechanism accounting for the association between MS and high AFP levels may be a state of chronic low-level inflammation and oxidative stress.

Serum AFP is generally recognized as an important tumor marker and has specific diagnostic utilities [30]. Continuous elevation of AFP levels up to the pathological range in adults has been associated with hepatocellular carcinoma, gastric cancer, hepatic necrosis, hepatic cirrhosis, acute hepatitis, chronic active hepatitis, ataxia telangiectasia, Wiskott-Aldrich syndrome, and pregnancy [30, 31]. Continuous elevation of serum AFP has rarely been found in participants with no obvious pathology [30, 32].

Adult hepatocytes re-express AFP mainly through three mechanisms: (i) Adult hepatocytes are regarded as functional stem cells and have the inherent capacity for regeneration [33]. When hepatocytes regenerate, AFP levels increase [34]. Hepatocyte proliferation during reparative and regenerating growth is associated with progenitor cell activation and is revealed by observation of rising serum AFP levels, cellular AFP immune expression, and AFP gene expression during hepatocyte division in liver regeneration after chemical injury or partial hepatectomy [33-36]. (ii) DNA damage to hepatocytes induced by oxidative stress results in the activation of transcription factors and induces the expression of proto-oncogenes by DNA methylation, leading to genomic instability and, consequently, hepatocarcinogenesis. Additionally, hepatomas that originate from spontaneously retro-differentiated hepatocytes can express AFP [27, 37, 38]; (c) In very extensive or chronic inflammation liver injury models, when the regenerative capacity of hepatocytes is impeded, reconstitution of the liver occurs through biliary epithelial cells (oval cells) that possess regenerative capacity with multilinear differentiation potential. Biliary epithelial cell proliferation leads to increases in AFP-specific immune expression [34, 39, 40].

Recently, a large sample cross-sectional study by $\mathrm{Xu}$ et al. [16] found that participants with FLD had higher AFP levels than those without FLD, and suggested that hepatocyte necrosis and subsequent hepatic regeneration may be responsible for the elevation of serum AFP levels. Babalı et al. [12] found that patients with NAFLD had higher AFP levels, and suggested that hepatic inflammation, regeneration and/or fibrosis may be responsible for the elevation of serum AFP levels in patients with severe fatty liver. We also considered it possible that hepatocyte steatosis and subsequent hepatic regeneration are responsible for the elevation of serum AFP levels in patients with MS. Hepatic steatosis has been reported to be a common histological feature of hepatitis $\mathrm{C}$ viral infection [41], and Goldstein et al. [42] found altered hepatocyte-hepatocyte interaction and loss of normal architectural arrangements leading to the elevation of serum AFP levels in patients with chronic hepatitis.

MS is a lifestyle and diet-related chronic noncommunicable disease that has become a major burden on global healthcare [38, 43, 44]. Epidemiological studies indicate that nutrition plays a very important role in the development and progression of MS. A diet high in fat, cholesterol, and sugar promotes the redistribution of body fat from peripheral to visceral adipose tissue and affects total body weight $[43,44]$. Animal experiments have been reported in which liver tissue sections from 
rats with diet-induced MS showed increased wet weight, hepatocyte damage, fat vacuoles, fibrosis, collagen deposition, ballooning, and inflammatory cell infiltration $[45,46]$. Body fat mass was found to increase in these studies, and impaired glucose tolerance, plasma lipid abnormalities, hyperinsulinemia, and increased liver enzyme activity were observed $[45,46]$.

Oxidative stress has emerged as a central player in some chronic metabolic diseases and leads to the oxidation of lipids, proteins, and nucleic acids. The above-mentioned pathological liver changes and stages of fibrosis were also found to be associated with oxidative DNA damage $[27,38]$. Nishida N et al. found that patients with high serum AFP levels and high degrees of ballooning and inflammatory infiltration had an accumulation of oxidative DNA damage [38]. Furukawa et al. [29] found that increased oxidative stress led to dysregulated production of adipocytokines, and that increased reactive oxygen species production from adipose tissue led to increased oxidative stress in the blood, which hazardously affected the liver, skeletal muscle, and the aorta in MS patients. Kuhlmann et al. and Assimakopoulos et al. observed oval cell proliferation during liver regeneration concomitant with increased AFP expression, with the mechanism being different from AFP expression in hepatocytes $[34,39]$. From this previous research and our own observations, we considered that oxidative stress and oval cell proliferation were responsible for the elevation of serum AFP levels in patients with MS.

Our study had some limitations. First, serum insulin levels were not analyzed and homeostasis model of assessment-insulin resistance was not calculated. Our study indirectly indicates a relationship between serum AFP levels and IR, but further research on a direct relationship between AFP and IR needs to be conducted. Second, hepatic inflammation is an important factor in the progression of chronic liver disease and is regulated by chemokines [47]. We did not analyze the effect of chemokines in the relationship between serum AFP levels and IR. Third, our study was a cross-sectional study and no definitive conclusions can be made on causality or temporal associations between AFP and MS.

\section{Conclusion}

In conclusion, the results from this study suggest that participants with MS have significantly higher AFP levels than those without MS. Serum AFP levels were significantly associated with the prevalence of MS, though the linking of serum AFP levels and the prevalence of MS is complicated and requires further prospective investigation.

\section{Ethical approval and consent to participate}

Informed consent was obtained from all participants and the study was approved by the ethics committee of the First Affiliated Hospital of the Medical College of Zhejiang University of China.

\begin{abstract}
Abbreviations
AFP: alpha-fetoprotein; ALT: alanine aminotransferase; AST: aspartate aminotransferase; $\mathrm{Cr}$ : creatinine; DPB: diastolic blood pressure; FLD: fatty liver disease; FPG: fasting plasma glucose; HbA1c: hemoglobin A1c; HDL-c: high-density lipoprotein cholesterol; IR: insulin resistance; LDL-c: lowdensity lipoprotein cholesterol; MS: metabolic syndrome; NAFLD: non-alcoholic fatty liver disease; OR: odds ratio; SPB: systolic blood pressure; Tch: total cholesterol; TG: triglycerides; WC: waist circumference; $\gamma$-GT: gammaglutamyltransferase; $95 \% \mathrm{Cl}$ : $95 \%$ confidence interval.
\end{abstract}

\section{Competing interests}

The authors declare that they have no competing interests.

Authors' contributions

GF designed the experiments. YC, YZ, LF, and JZ performed the experiments. $Y C, Y Z$, and $J Z$ wrote the main manuscript. $Y Z$ revised the manuscript. All authors read and approved the final manuscript.

\section{Acknowledgements}

This work was financially supported by grants from the Zhejiang Provincial Natural Science Foundation of China (LY15H190002) and the Science Foundations of Health Bureau of Zhejiang Province (2013KYB116 and 2014KYA252).

\section{Author details}

'Department of Clinical Laboratory, Zhejiang Provincial Hospital of Traditional Chinese Medicine, The First Affiliated Hospital of Zhejiang Chinese Medical University, Youdian Road \#54, Hangzhou 310006, China. ${ }^{2}$ Department of Clinical Laboratory, The First Affiliated Hospital of Zhejiang University School of Medicine, Qingchun Road \#79, Hangzhou 310003, China. ${ }^{3}$ The Affiliated Women's Hospital of Zhejiang University School of Medicine, Xueshi Road \#1, Hangzhou 310006, China.

Received: 9 December 2015 Accepted: 24 April 2016

Published online: 27 April 2016

References

1. Hong AR, Lim S. Clinical characteristics of metabolic syndrome in Korea, and its comparison with other Asian countries. J Diab Investig. 2015;6(5):508-15.

2. Mayans L. Metabolic Syndrome: Insulin Resistance and Prediabetes. FP Essent. 2015:435:11-6.

3. Bragg DA, Walling A. Metabolic Syndrome: Hyperlipidemia. FP Essent. 2015; 435:17-23.

4. Tie HT, Shi R, Li ZH, Zhang M, Zhang C, Wu QC. Risk of major adverse cardiovascular events in patients with metabolic syndrome after revascularization: A meta-analysis of eighteen cohorts with 18457 patients. Metabolism. 2015;64(10):1224-34

5. Williams T. Metabolic Syndrome. Nonalcoholic Fatty Liver Dis FP Essent. 2015:435:24-9.

6. Gu D, Reynolds K, Wu X, Chen J, Duan X, Reynolds RF, Whelton PK, He J, InterASIA Collaborative Group. InterASIA Collaborative Group. Prevalence of the metabolic syndrome and overweight among adults in China. Lancet. 2005;365(9468):1398-405.

7. Zhou HC, Lai YX, Shan ZY, Jia WP, Yang WY, Lu JM, Weng JP, Ji LN, Liu J, Tian HM, Ji QH, Zhu da L, Chen L, Guo XH, Zhao ZG, Li Q, Zhou ZG, Ge JP, Shan GL. Effectiveness of different waist circumference cut-off values in predicting metabolic syndrome prevalence and risk factors in adults in China. Biomed Environ Sci. 2014;27(5):325-34.

8. Welty FK, Alfaddagh A, Elajami TK. Targeting inflammation in metabolic syndrome. Transl Res. 2015;S1931-5244(15):00222-4.

9. Takikawa Y, Suzuki K. Is AFP a new reliable marker of liver regeneration in acute hepatic failure? J Gastroenterol. 2002;37(8):681-2.

10. Yachnin S. The clinical significance of human alpha-fetoprotein. Ann Clin Lab Sci. 1978:8(2):84-90. 
11. Crandall BF. Alpha-fetoprotein: a review. Crit Rev Clin Lab Sci. 1981;15(2): 127-85.

12. Babalı A, Cakal E, Purnak T, Bıyıkoğlu I, Cakal B, Yüksel O, Köklü S.. Serum a-fetoprotein levels in liver steatosis. Hepatol Int. 2009;3(4):551-5.

13. Wu JT. Serum alpha-fetoprotein and its lectin reactivity in liver diseases: a review. Ann Clin Lab Sci. 1990:20(2):98-105.

14. Qin LX, Tang ZY. Recent progress in predictive biomarkers for metastatic recurrence of human hepatocellular carcinoma: a review of the literature. J Cancer Res Clin Oncol. 2004;130(9):497-513.

15. Wong RJ, Ahmed A, Gish RG. Elevated alpha-fetoprotein: differential diagnosis - hepatocellular carcinoma and other disorders. Clin Liver Dis. 2015;19(2):309-23.

16. Xu P, Xu CF, Wan XY, Yu CH, Shen C, Chen P, Xu GY, Li YM. Association between serum alpha-fetoprotein levels and fatty liver disease: a crosssectional study. World J Gastroenterol. 2014;20(33):11865-70.

17. Shao JH, Jin YL, Yu LL, Shen X, Li DX, Wang K. study on the application and evaluation of different diagnostic criteria for metabolic syndrome in community people. Chin J Diab. 2009;17(11):801-3.

18. Verhagen SN, Wassink AM, van der Graaf Y, Gorter PM, Visseren FL. SMART Study Group. Insulin resistance increases the occurrence of new cardiovascular events in patients with manifest arterial disease without known diabetes. the SMART study. Cardiovasc Diabetol. 2011;10:100.

19. Gallagher EJ, Leroith D, Karnieli E. Insulin resistance in obesity as the underlying cause for the metabolic syndrome. Mt Sinai J Med. 2010;77(5):511-23.

20. Porte Jr D, Kahn SE. Beta-cell dysfunction and failure in type 2 diabetes: potential mechanisms. Diabetes. 2001;50 Suppl 1:S160-163.

21. Kelley DE, McKolanis TM, Hegazi RA, Kuller LH, Kalhan SC. Fatty liver in type 2 diabetes mellitus: relation to regional adiposity, fatty acids, and insulin resistance. Am J Physiol Endocrinol Metab. 2003;285(4):E906-16.

22. Kotronen A, Yki-Jarvinen H. Fatty liver: a novel component of the metabolic syndrome. Arterioscler Thromb Vasc Biol. 2008;28(1):27-38.

23. Fabbrini E, Magkos F. Hepatic Steatosis as a Marker of Metabolic Dysfunction. Nutrients. 2015;7(6):4995-5019.

24. Porepa L, Ray JG, Sanchez-Romeu P, Booth GL. Newly diagnosed diabetes mellitus as a risk factor for serious liver disease. CMAJ. 2010; 182(11):E526-531.

25. Matsuzaka T, Shimano H. Molecular mechanisms involved in hepatic steatosis and insulin resistance. J Diab Investig. 2011:2(3):170-5.

26. Desai M, Jellyman JK, Ross MG. Epigenomics, gestational programming and risk of metabolic syndrome. Int J Obes (Lond). 2015:39(4):633-41.

27. Rani V, Deep G, Singh RK, Palle K, Yadav UC. Oxidative stress and metabolic disorders: Pathogenesis and therapeutic strategies. Life Sci. 2016;148:183-93.

28. Furukawa S, Fujita T, Shimabukuro M, Iwaki M, Yamada Y, Nakajima Y, Nakayama O, Makishima M, Matsuda M, Shimomura I. Increased oxidative stress in obesity and its impact on metabolic syndrome. J Clin Invest. 2004; 114(12):1752-61.

29. Bonomini F, Rodella LF, Rezzani R. Metabolic syndrome, aging and involvement of oxidative stress. Aging Dis. 2015;6(2):109-20.

30. Yuen MF, Lai CL. Serological markers of liver cancer. Best Pract Res Clin Gastroenterol. 2005;19(1):91-9.

31. Baig JA, Alam JM, Mahmood SR, Baig M, Shaheen R, Sultana I, Waheed A. Hepatocellular carcinoma (HCC) and diagnostic significance of A-fetoprotein (AFP). J Ayub Med Coll Abbottabad. 2009;21(1):72-5.

32. Bloomer JR, Waldmann TA, Mclntire KR, Klatskin G. alpha-fetoprotein in noneoplastic hepatic disorders. JAMA. 1975:233:38-41.

33. Fausto N. Liver regeneration. J Hepatol. 2000;32(1 Suppl):19-31.

34. Kuhlmann WD, Peschke P. Hepatic progenitor cells, stem cells, and AFP expression in models of liver injury. Int J Exp Pathol. 2006;87(5):343-59.

35. Dabeva MD, Laconi E, Oren R, Petkov PM, Hurston E, Shafritz DA. Liver regeneration and alpha-fetoprotein messenger RNA expression in the retrorsine model for hepatocyte transplantation. Cancer Res. 1998;58(24): 5825-34.

36. Sell S, Becker FF, Leffert HL, Watabe L. Expression of an oncodevelopmental gene product (alpha-fetoprotein) during fetal development and adult oncogenesis. Cancer Res. 1976;36(11 Pt. 2):4239-49.

37. Farber E, Sarma DS. Hepatocarcinogenesis: a dynamic cellular perspective. Lab Invest. 1987;56(1):4-22.

38. Nishida N, Yada N, Hagiwara S, Sakurai T, Kitano M, Kudo M. Unique features associated with hepatic oxidative DNA damage and DNA methylation in non-alcoholic fatty liver disease. J Gastroenterol Hepatol. 2016; doi: 10.1111/ jgh.13318
39. Assimakopoulos SF, Tsamandas AC, Alexandris IH, Georgiou C, Vagianos CE, Scopa CD. Stimulation of oval cell and hepatocyte proliferation by exogenous bombesin and neurotensin in partially hepatectomized rats. World J Gastrointest Pathophysiol. 2011;2(6):146-54.

40. Alison MR, Golding MH, Sarraf CE. Pluripotential liver stem cells: facultative stem cells located in the biliary tree. Cell Prolif. 1996;29(7):373-402.

41. Hu KQ, Currie SL, Shen H, Cheung RC, Ho SB, Bini EJ, McCracken JD, Morgan T, Bräu N, Schmidt WN, et al. VA HCV-001 Study Group: Clinical implications of hepatic steatosis in patients with chronic hepatitis C: a multicenter study of U.S. veterans. Dig Dis Sci. 2007;52(2):570-8.

42. Goldstein NS, Blue DE, Hankin R, Hunter S, Bayati N, Silverman AL, Gordon SC. Serum alpha-fetoprotein levels in patients with chronic hepatitis $C$. Relationships with serum alanine aminotransferase values, histologic activity index, and hepatocyte MIB-1 scores. Am J Clin Pathol. 1999:111(6):811-6.

43. Roche HM, Phillips C, Gibney MJ. The metabolic syndrome: the crossroads of diet and genetics. Proc Nutr Soc. 2005;64(3):371-7.

44. Perez-Martinez P, Garcia-Rios A, Delgado-Lista J, Perez-Jimenez F, Lopez-Miranda J. Metabolic syndrome: evidences for a personalized nutrition. Mol Nutr Food Res. 2012;56(1):67-76.

45. Iyer A, Kauter K, Alam MA, Hwang SH, Morisseau C, Hammock BD, Brown L. Pharmacological inhibition of soluble epoxide hydrolase ameliorates diet-induced metabolic syndrome in rats. Exp Diabetes Res. 2012;2012:758614

46. Panchal SK, Poudyal H, lyer A, Nazer R, Alam MA, Diwan V, Kauter K, Sernia C, Campbell F, Ward L, Gobe G, Fenning A, Brown L. High-carbohydrate, high-fat diet-induced metabolic syndrome and cardiovascular remodeling in rats. J Cardiovasc Pharmacol. 2011:57(5):611-24.

47. Marra F, Tacke F. Roles for chemokines in liver disease. Gastroenterology. 2014;147(3):577-94.

\section{Submit your next manuscript to BioMed Central and we will help you at every step:}

- We accept pre-submission inquiries

- Our selector tool helps you to find the most relevant journal

- We provide round the clock customer support

- Convenient online submission

- Thorough peer review

- Inclusion in PubMed and all major indexing services

- Maximum visibility for your research

Submit your manuscript at www.biomedcentral.com/submit
C Biomed Central 